\title{
Geometric Description of the Thermodynamics of the Noncommutative Schwarzschild Black Hole
}

\author{
Alexis Larrañaga, ${ }^{1}$ Natalia Herrera, ${ }^{2}$ and Juliana Garcia ${ }^{2}$ \\ ${ }^{1}$ National Astronomical Observatory, National University of Colombia, Bogotá 11001000, Colombia \\ ${ }^{2}$ Department of Physics, National University of Colombia, Bogotá 11001000, Colombia
}

Correspondence should be addressed to Alexis Larrañaga; ealarranaga@unal.edu.co

Received 13 March 2013; Accepted 8 May 2013

Academic Editor: Rong-Gen Cai

Copyright ( 2013 Alexis Larrañaga et al. This is an open access article distributed under the Creative Commons Attribution License, which permits unrestricted use, distribution, and reproduction in any medium, provided the original work is properly cited.

\begin{abstract}
The thermodynamics of the noncommutative Schwarzschild black hole is reformulated within the context of the recently developed formalism of geometrothermodynamics (GTD). Using a thermodynamic metric which is invariant with respect to Legendre transformations, we determine the geometry of the space of equilibrium states and show that phase transitions, which correspond to divergencies of the heat capacity, are represented geometrically as singularities of the curvature scalar. This further indicates that the curvature of the thermodynamic metric is a measure of thermodynamic interaction.
\end{abstract}

\section{Introduction}

The work of Hawking [1] gives rise to an extensive study of the thermodynamics of black holes. One of the most interesting aspects of such study is the notion of critical behaviour that has arisen in several contexts, for example, Hawking and Page's [2] phase transition in AdS space and the idea that the extremal limit of different black hole families might themselves be regarded as genuine critical points [3-6]. A complete explanation for the final state of the black hole after the evaporation is important, but it has not been achieved, presumably because there is not yet a full quantum gravity theory. Today, one of the strongest candidates for quantum gravity is string theory, in which coordinates of the target spacetime become noncommuting operators on a D-brane as

$$
\left[x^{\mu}, x^{\nu}\right]=i \theta^{\mu \nu},
$$

where $\theta^{\mu \nu}$ is an antisymmetric matrix which determines the fundamental cell discretization of spacetime in the same way as the Planck constant discretizes the phase space. It has also been shown that Lorentz invariance and unitary can be achieved by assuming $\theta^{\mu \nu}=\theta \operatorname{diag}\left(\epsilon_{1}, \ldots, \epsilon_{n / 2}\right)$, where $n$ is the dimension of spacetime, and $\sqrt{\theta}$ is a constant that provides a minimum scale. A noncommutative static and spherically symmetric black hole solution whose commutative limit is the Schwarzschild metric has been found in [7-11]. The thermodynamics and evaporation process of this black hole have been studied in [12], while the entropy issue is discussed in $[13,14]$ and its Hawking radiation in [15].

On the other hand, the use of geometry in statistical mechanics was pioneered by Ruppeiner $[16,17]$ and Weinhold [18, 19], who suggested that the curvature of a metric defined on the space of parameters of a statistical mechanical theory could provide information about the phase structure. However, when these methods are applied to the study of black hole thermodynamics, some puzzling anomalies appear. A possible solution to these issues was suggested by Quevedo's geometrothermodynamics (GTD), whose starting point [20] was the observation that standard thermodynamics was invariant with respect to Legendre transformations, and an interesting aspect in this formalism is that it indicates that phase transitions occur at those points where the thermodynamic curvature is singular.

In this paper, we apply the GTD formalism to the noncommutative Schwarzschild black hole to investigate the behaviour of the thermodynamical curvature in the search of phase transitions. This noncommutative black hole has two horizons and an evaporation process that ends up in a extremal zero-temperature configuration. Thus, similar evaporation process and thermodynamical properties when 
compared with the behaviour of Reissner-Nordström black hole are expected as stressed in $[21,22]$. Since the noncommutative Schwarzschild black hole is described by the thermodynamical variables mass, temperature, and entropy, the space of equilibrium thermodynamic states has just two dimensions, making it impossible to analyse the phase transitions structure from the curvature scalar. Therefore, we need to treat the noncommutative parameter $\theta$ as a thermodynamical variable, playing a similar role to that of the electric charge in Reissner-Nordström solution. This consideration can be justified from the point of view of the quantum fluctuations of geometry which are naturally expected to arise from the noncommutativity of spacetime.

\section{Geometrothermodynamics in Brief}

Let $\mathscr{T}$ be the $(2 n+1)$-dimensional thermodynamic phase space with coordinates given by the thermodynamic potential $\Phi$, the extensive variables $E^{a}$, and the intensive variables $I^{a}$. These coordinates will be noted as $Z^{A}=\left\{\Phi, E^{a}, I^{a}\right\}$ with $a=1, \ldots, n$. We define on $\mathscr{T}$ a nondegenerate metric $G=G\left(Z^{A}\right)$ and Gibbs 1-form $\Theta=d \Phi-\delta_{a b} I^{a} d E^{b}$. If the condition $\Theta \wedge(d \Theta)^{n} \neq 0$ is satisfied, the $\operatorname{set}(\mathscr{T}, \Theta, G)$ is called a contact Riemannian manifold. Gibbs 1-form is invariant with respect to Legendre transformations, while the metric $G$ is Legendre invariant if its functional dependence on $Z^{A}$ does not change under a Legendre transformation. Following the GTD formalism, we will impose this invariance in order to guarantee that the geometric properties of $G$ do not depend on the thermodynamic potential used for its construction. We introduce the $n$-dimensional subspace $\mathscr{E} \subset \mathscr{T}$ called the space of equilibrium thermodynamic states through the following smooth mapping:

$$
\begin{gathered}
\varphi: \mathscr{E} \longrightarrow \mathscr{T}, \\
\left(E^{a}\right) \longrightarrow\left(\Phi, E^{a}, I^{a}\right),
\end{gathered}
$$

with $\Phi=\Phi\left(E^{a}\right)$ and subject to the condition $\varphi^{*}(\Theta)=0$ which gives the following relations:

$$
\begin{gathered}
d \Phi=\delta_{a b} I^{a} d E^{b} \\
\frac{\partial \Phi}{\partial E^{a}}=\delta_{a b} I^{b}
\end{gathered}
$$

Equation (3) corresponds to the first law of thermodynamics, whereas (4) is usually known as the condition for thermodynamic equilibrium, that is, the intensive thermodynamic variables are dual to the extensive ones. The second law of thermodynamics is equivalent to the convexity condition on the thermodynamic potential,

$$
\frac{\partial^{2} \Phi}{\partial E^{a} \partial E^{b}} \geq 0
$$

The mapping $\varphi$ implies that the equation $\Phi=\Phi\left(E^{a}\right)$, known as the fundamental equation, must be explicitly given and from this relation all the thermodynamical information can be derived. The potential satisfies the homogeneity condition $\Phi\left(\lambda E^{a}\right)=\lambda^{\beta} \Phi\left(E^{a}\right)$, with $\lambda$ and $\beta$ constant parameters, so it also satisfies Euler's identity as follows:

$$
\beta \Phi\left(E^{a}\right)=\delta_{a \mathrm{~b}} I^{b} E^{a}
$$

Using the first law of thermodynamics, this equation becomes Gibbs-Duhem relation:

$$
(1-\beta) \delta_{a b} I^{a} d E^{b}+\delta_{a b} E^{a} d I^{b}=0 .
$$

A thermodynamic system is described by a thermodynamic metric $G$ if it is invariant with respect to transformations which do not modify the contact structure of $\mathscr{T}$. In particular, $G$ must be invariant with respect to Legendre transformations in order for GTD to describe thermodynamic properties in terms of geometric concepts. A Legendre invariant metric $G$ induces a Legendre invariant, nondegenerate metric structure $g$ on $\mathscr{E}$ through the pullback $\varphi^{*}$ as $g=\varphi^{*}(G)[20]$.

The results of Quevedo et al. [23-25] showed that if the curvature of the thermodynamic metric is to be considered as a measure of the thermodynamic interaction, this metric should be flat only for systems with no thermodynamic interaction. Hence, phase transitions must occur at those points where the thermodynamic curvature is singular. There is a vast number of metrics on $\mathscr{T}$ that satisfy the Legendre invariance condition, and some results seem to show that the metric structure of the phase manifold determines the type of systems that can be described by a specific thermodynamic metric. For instance, a pseudo-Euclidean structure of the form

$$
G=\Theta^{2}+\left(\delta_{a b} E^{a} I^{b}\right)\left(\eta_{c d} d E^{c} d I^{d}\right)
$$

with $\eta_{c d}=\operatorname{diag}(-1,1,1, \ldots, 1)$ is Legendre invariant and induces the following metric:

$$
g=\left(E^{f} \frac{\partial \Phi}{\partial E^{f}}\right)\left(\eta_{a b} \delta^{b c} \frac{\partial^{2} \Phi}{\partial E^{c} \partial E^{d}} d E^{a} d E^{d}\right),
$$

which appears to describe thermodynamical systems characterized with second-order phase transitions.

\section{The Noncommutative Schwarzschild Black Hole}

In a commutative space, the mass density of a point particle is expressed as a product of its mass with the Dirac delta function, but in a noncommutative space, it is expected that such a description of point mass is not possible due to the fuzziness of space which arises as a consequence of position-position uncertainty relation. To introduce the noncommutative correction in the expression of mass density, we replace the Dirac delta function by a Gaussian distribution of minimal width $\sqrt{\theta}$,

$$
\rho_{\theta}=\frac{M}{(4 \pi \theta)^{3 / 2}} e^{-r^{2} / 4 \theta},
$$


where the noncommutativity parameter $\theta$, which defines the minimum scale, is considered to be a small positive number. Consequently, the mass of the black hole can be determined by integrating (10) over a volume of radius $r$,

$$
m_{\theta}(r)=\int_{0}^{r} 4 \pi r^{\prime 2} \rho_{\theta}\left(r^{\prime}\right) d r^{\prime}=\frac{2 M}{\sqrt{\pi}} \gamma\left(\frac{3}{2}, \frac{r^{2}}{4 \theta}\right),
$$

where $\gamma\left(3 / 2, r^{2} / 4 \theta\right)$ is the lower incomplete gamma function,

$$
\gamma(a, z)=\int_{0}^{z} t^{a-1} e^{-t} d t
$$

In the commutative limit, $\theta \rightarrow 0, \gamma\left(3 / 2, r^{2} / 4 \theta\right)$ becomes the usual gamma function $\Gamma(3 / 2)$ and $m_{\theta}(r) \rightarrow M$. Substituting this result in the mass term of the Schwarzschild's solution, we obtain the noncommutative Schwarzschild metric,

$$
\begin{aligned}
d s^{2}= & -\left(1-\frac{4 M}{r \sqrt{\pi}} \gamma\left(\frac{3}{2}, \frac{r^{2}}{4 \theta}\right)\right) d t^{2} \\
& +\left(1-\frac{4 M}{r \sqrt{\pi}} \gamma\left(\frac{3}{2}, \frac{r^{2}}{4 \theta}\right)\right)^{-1} d r^{2}+r^{2} d \Omega^{2} .
\end{aligned}
$$

The classical Schwarzschild's metric is obtained from (13) in the limit $r / \sqrt{\theta} \rightarrow \infty$, and event horizon(s) can be found at points where $g_{00}\left(r_{H}\right)=0$, which corresponds to the following condition:

$$
r_{H}=\frac{4 M}{\sqrt{\pi}} \gamma\left(\frac{3}{2}, \frac{r_{H}^{2}}{4 \theta}\right)
$$

The analysis of (14) determines that, instead of a singleevent horizon, there are three different possibilities depending on a critical mass $M_{0}=1.9 \sqrt{\theta},[9]$ :

(1) two distinct horizons for $M>M_{0}$,

(2) one degenerate horizon at $r_{0}=3.0 \sqrt{\theta}$, when $M=M_{0}$ (corresponds to the extremal black hole),

(3) no horizon for $M<M_{0}$.

Equation (14) can be conveniently rewritten in terms of the gamma function as

$$
r_{H}=2 M\left[1-\frac{2}{\sqrt{\pi}} \Gamma\left(\frac{3}{2}, \frac{M^{2}}{\theta}\right)\right] \text {, }
$$

where the first term in the right hand side is the Schwarzschild radius, while the second term brings in noncommutative corrections. The area of the event horizon can be written as

$$
A=4 \pi r_{H}^{2}=16 \pi M^{2}\left[1-\frac{2}{\sqrt{\pi}} \Gamma\left(\frac{3}{2}, \frac{M^{2}}{\theta}\right)\right]^{2}
$$

and, hence, the entropy associated with the black hole is simply

$$
S=\frac{A}{4}=4 \pi M^{2}\left[1-\frac{2}{\sqrt{\pi}} \Gamma\left(\frac{3}{2}, \frac{M^{2}}{\theta}\right)\right]^{2} .
$$

The Hawking temperature may be calculated as usual, giving the temperature of Schwarzschild's black hole plus a correction term,

$$
T=\left.\frac{1}{4 \pi} \frac{d g_{00}}{d r}\right|_{r=r_{H}}=\frac{1}{4 \pi r_{H}}\left[1-\frac{r_{H}^{3}}{4 \theta^{3 / 2}} \frac{e^{-r_{H}^{2} / 4 \theta}}{\gamma\left(3 / 2, r_{H}^{2} / 4 \theta\right)}\right] .
$$

As is well known, in the commutative case, the temperature diverges, putting a limit on the validity of the conventional description of Hawking radiation. However, the temperature obtained in (18) includes noncommutative effects which are relevant at small distances, making $T$ to deviate from the standard hyperbola and, instead of diverge, it reaches a maximum value at the radius $r_{c} \simeq 4.7 \sqrt{\theta}$ or correspondingly at the mass $M_{c} \simeq 2.4 \sqrt{\theta}$ and then quickly drops to zero temperature for $r_{H}=r_{0}$, which corresponds to the radius of the extremal black hole. This behaviour of the evaporation precession is typical of black holes with two horizons as, for example, Reissner-Nordström's solution.

From (14), we may write

$$
M=\frac{\sqrt{S}}{4 \gamma(3 / 2, S / 4 \pi \theta)},
$$

which may be considered as the fundamental thermodynamical equation $M=M(S, \theta)$ which relates the total energy of the black hole, $M$, with the extensive variables, entropy, and noncommutativity parameter, and from which all the thermodynamical information can be derived. The inclusion of the parameter $\theta$ as a thermodynamical variable is justified from the analogy between the noncommutative Schwarzschild's black hole and the Reissner-Nordström solution which has been commented in [21] and studied extensively in [22], where the authors showed that the noncommutativity parameter plays a similar role with the electric charge. Even more, the authors showed that the thermodynamical properties and the evaporation process are similar in both solutions.

In the geometric formulation of thermodynamics, we will choose the extensive variables as $E^{a}=\{S, \theta\}$ and the corresponding intensive variables as $I^{a}=\{T, \Psi\}$, where $T$ is the temperature, and $\Psi$ is the generalised variable conjugate to the state parameter $\theta$. Therefore, the coordinates that we will use in the 5-dimensional thermodynamical space $\mathscr{T}$ are $Z^{A}=\{M, S, \theta, T, \Psi\}$. The contact structure of $\mathscr{T}$ is generated by the 1 -form,

$$
\Theta=d M-T d S-\Psi d \theta .
$$

To obtain the induced metric in the space of equilibrium states $\mathscr{E}$, we will introduce the following smooth mapping:

$$
\varphi:\{S, \theta\} \longmapsto\{M(S, \theta), S, \theta, T(S, \theta), \Psi(S, \theta)\}
$$


along with the condition $\varphi^{*}(\Theta)=0$ that corresponds to the first law $d M=T d S+\Psi d \theta$. The conjugate variables to $S$ and $\theta$ are evaluated as

$$
\begin{gathered}
T=\frac{\partial M}{\partial S} \\
=\frac{1}{8 \sqrt{S} \gamma(3 / 2, S / 4 \pi \theta)}\left[1-\frac{1}{4} \sqrt{\left(\frac{S}{\pi \theta}\right)^{3}} \frac{e^{-S / 4 \pi \theta}}{\gamma(3 / 2, S / 4 \pi \theta)}\right], \\
\Psi=\frac{\partial M}{\partial \theta}=\frac{S^{2}}{32 \sqrt{\pi^{3} \theta^{5}}} \frac{e^{-S / 4 \pi \theta}}{\gamma^{2}(3 / 2, S / 4 \pi \theta)} .
\end{gathered}
$$

Using (8), $\mathscr{T}$ becomes a Riemannian manifold by defining

$$
G=(d M-T d S-\Psi d \theta)^{2}+(S T+\Psi \theta)(-d S d T+d \Lambda d \theta) .
$$

This metric has nonzero curvature, and its determinant is $\operatorname{det}[G]=(S T+\Psi \theta)^{4} / 16$. To obtain the induced metric in the space of equilibrium states $\mathscr{E}$, we use (9), obtaining

$$
g=\left(S M_{S}+\theta M_{\theta}\right)\left(\begin{array}{cc}
-M_{S S} & 0 \\
0 & M_{\theta \theta}
\end{array}\right)
$$

where subscripts represent partial derivative with respect to the corresponding coordinate. Clearly, the determinant of this metric is

$$
\operatorname{det}[g]=-M_{S S} M_{\theta \theta}\left(S M_{S}+\theta M_{\theta}\right)
$$

\section{Phase Transitions and the Curvature Scalar}

Phase transitions are an interesting subject in the study of black holes thermodynamics because there is no unanimity in their definition. As is well known, ordinary thermodynamics defines phase transitions by looking for singular points in the behaviour of thermodynamical variables. For example, Davies $[26,27]$ showed that divergences in the heat capacity

$$
C=T \frac{\partial S}{\partial T}=\frac{M_{S}}{M_{S S}}
$$

indicate phase transitions. From these arguments one can expect that phase transitions occur at $M_{S S}=0$. In geometrothermodynamics, the apparition of phase transitions is related with the divergences of the curvature scalar $R$ in the space of equilibrium states $\mathscr{E}$. This can be understood by remembering that $R$ always contains the determinant of the metric $g$ in the denominator, so that zeros of $\operatorname{det}[g]$ could lead to curvature singularities if those zeros are not canceled by the zeros of the numerator.

The metric given by (24) has the determinant (25) which is proportional to $S_{M M}$ and $S_{\theta \theta}$. This fact makes clear the coincidence with the divergence of the heat capacity. Even more, the curvature scalar $R$ for the metric $g$ has the denominator

$$
D=\left(S M_{S}+\theta M_{\theta}\right)^{3} M_{S S}^{2} M_{\theta \theta}^{2}
$$

which makes $R$ diverge when $S_{M M}=0$ or $S_{\theta \theta}=0$, whereas the numerator is a rather cumbersome expression that cannot be written in a compact form. From (19), we have

$$
\begin{aligned}
M_{S S}= & -\frac{1}{16 \sqrt{S^{3}} \gamma(3 / 2, S / 4 \pi \theta)} \\
& \times\left[1+\frac{1}{6} \sqrt{\left(\frac{S}{\pi \theta}\right)^{3} \frac{e^{-S / 4 \pi \theta}}{\gamma(3 / 2, S / 4 \pi \theta)}}\right. \\
& -\frac{1}{8} \sqrt{\left(\frac{S}{\pi \theta}\right)^{5} \frac{e^{-S / 4 \pi \theta}}{\gamma(3 / 2, S / 4 \pi \theta)}} \\
M_{\theta \theta}= & \left.\frac{1}{64 \sqrt{\pi^{3} \theta^{7}}}\left[\frac{S}{\pi \theta}\right)^{3} \frac{e^{-S / 2 \pi \theta}}{\gamma^{2}(3 / 2, S / 4 \pi \theta)}\right], \\
& \left.\times \frac{e^{-S / 4 \pi \theta}}{\gamma^{2}(3 / 2, S / 4 \pi \theta)} \cdot \frac{S \sqrt{S}}{10 \sqrt{\pi^{3} \theta^{3}}} \frac{e^{-S / 4 \pi \theta}}{\gamma(3 / 2, S / 4 \pi \theta)}\right]
\end{aligned}
$$

A numerical analysis shows that singularities in the curvature scalar $R$ appear exactly at the same points where the behaviour of the heat capacity indicates the presence of phase transitions (see Figure 1). In Figure 2, we show the particular phase transition for $\theta=0.5$ and located at the approximate value $S=57$. For all analysed values of the noncommutativity parameter $\theta$, it was obtained a similar behaviour, showing that, in fact, the points where phase transitions occur are characterised by curvature singularities of the thermodynamic metric.

\section{Conclusion}

Geometrothermodynamics is a differential geometry formalism whose objective is to describe in an invariant manner the properties of thermodynamic systems using geometric concepts. In this work, we reformulated the thermodynamics of the noncommutative Schwarzschild's black hole under the GTD formalism and considered the noncommutativity parameter $\theta$ as a new thermodynamical state variable. The total mass of the black hole is interpreted as its total energy, and the formalism gives a curvature scalar that diverges exactly at the point at which the heat capacity indicates the presence of a phase transition. Thus, we conclude that the curvature of the space of thermodynamic equilibrium states can be interpreted as a measure of the thermodynamic interaction.

These results clearly confirm that the phase manifold contains information about thermodynamic systems; however, a further exploration is necessary in order to understand how the thermodynamic information is encoded in the geometrical properties. For example, it is really interesting to address the problem of describing the black hole in an isolated cavity which is bigger than its Schwarzschild 


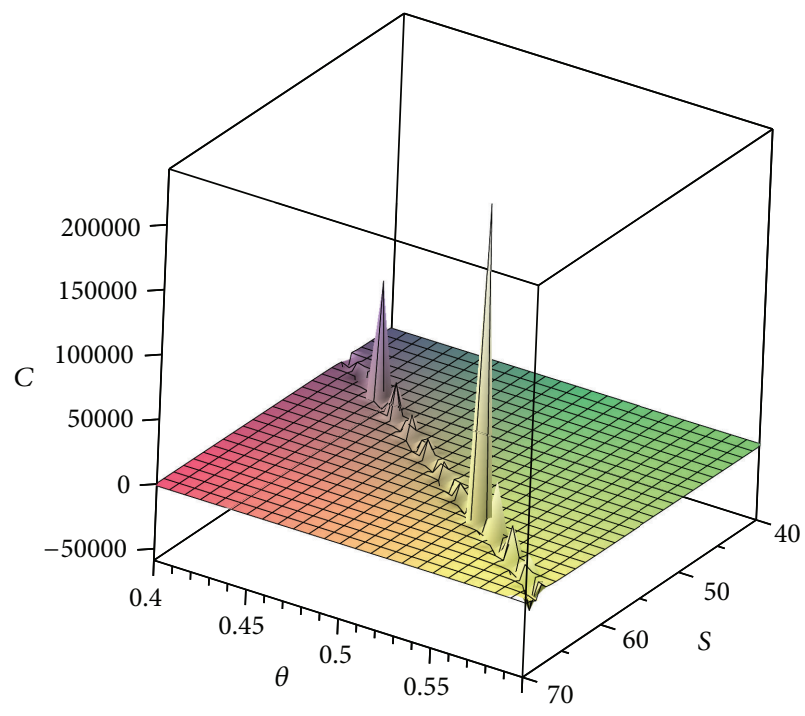

(a)

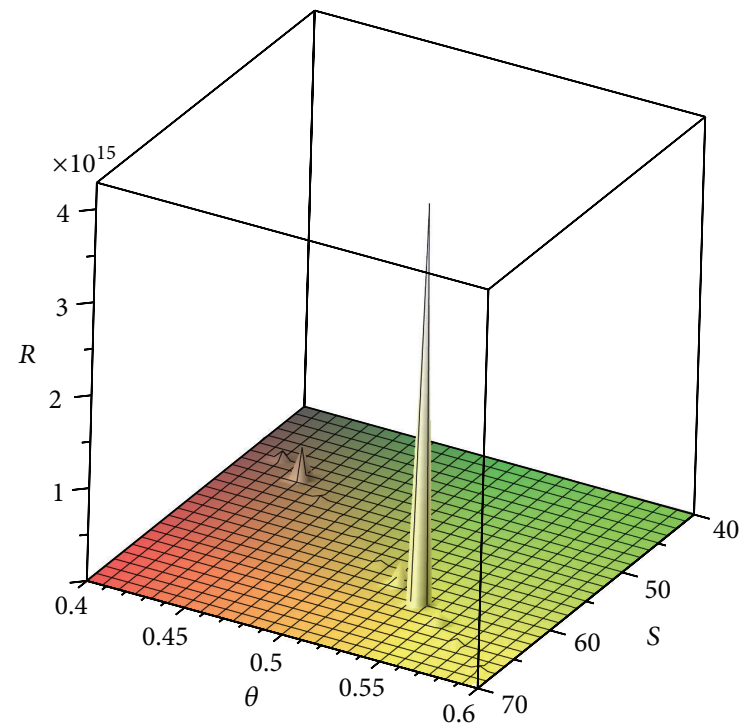

(b)

Figure 1: (a) Characteristic behaviour of the heat capacity as a function of $S$ and $\theta$. (b) Characteristic behaviour of the thermodynamic curvature scalar $R$ as a function of $S$ and $\theta$. Note that the singularities follow the same pattern in both functions, indicating the presence of phase transitions.

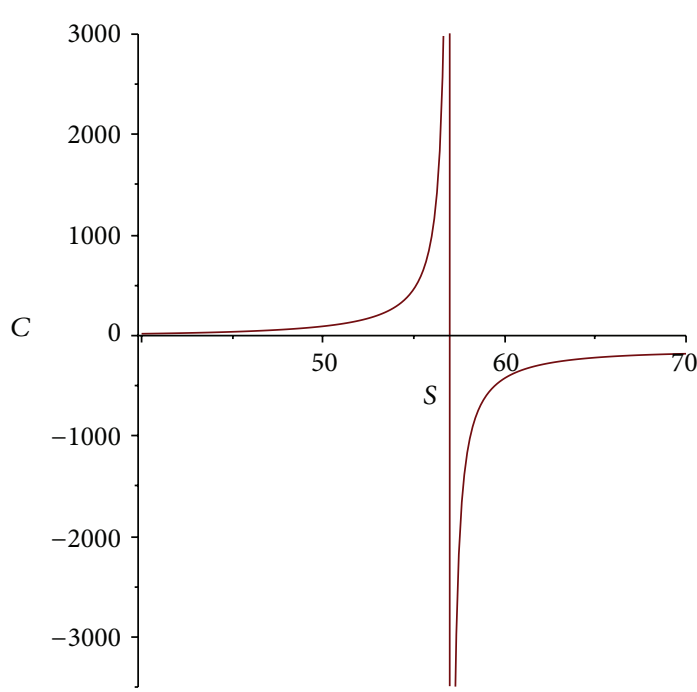

(a)

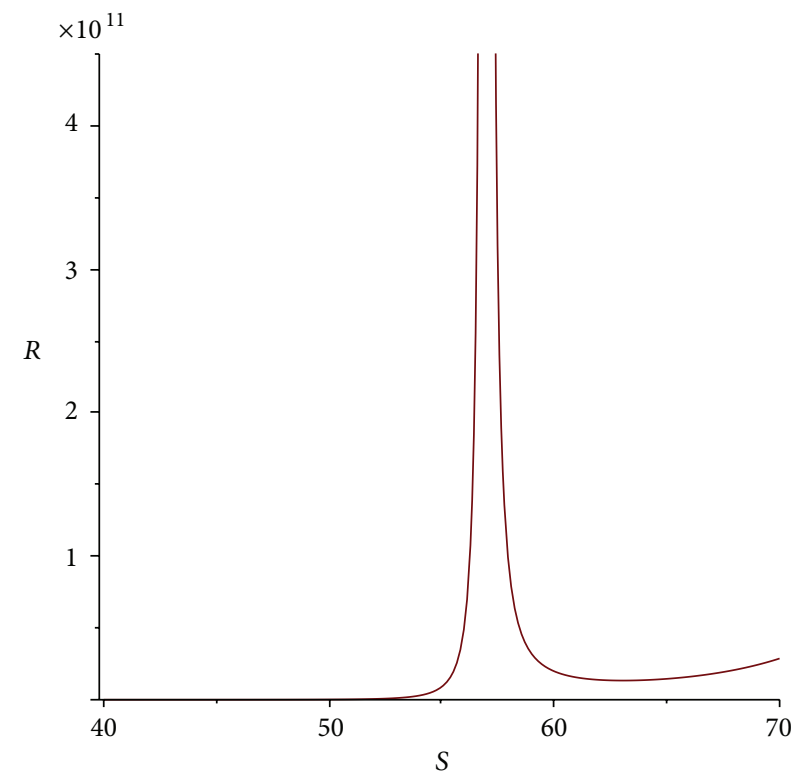

(b)

Figure 2: (a) Characteristic behaviour of the heat capacity as a function of the entropy $S$. The divergence indicates the point of a phase transition. (b) Behaviour of the thermodynamic curvature scalar $R$ as a function of the entropy $S$. The singularity is located at the point of the phase transition. In both figures, the noncommutativity parameter is set to $\theta=0.5$.

radius. As is known, the asymptotically flat solution has an unstable behaviour, because of the negative heat capacity; but when introducing the cavity, the temperature is fixed at a finite spatial boundary, and it is expected that the black hole reaches the thermodynamical equilibrium with the surrounding radiation if the total energy $E$ of the system is greater than some critical value $E_{c}$, depending on the volume of the cavity and the number of fields in the radiation [28].
It is well known that asymptotic flatness is not satisfied in reality and therefore, it is important to consider this situation in the specific case of the noncommutative black hole for which the heat capacity becomes positive for certain ranges of the horizon radius, making stable small and large black holes [22]. In a forthcoming paper, we will analyse how the GTD formalism describes this kind of system and how to implement the cavity in the geometric model. 


\section{Acknowledgment}

This work was supported by the Universidad Nacional de Colombia, Hermes Project Code 13038.

\section{References}

[1] S. W. Hawking, "Particle creation by black holes," Communications in Mathematical Physics, vol. 43, no. 3, pp. 199-220, 1975.

[2] S. W. Hawking and D. N. Page, "Thermodynamics of black holes in anti-de Sitter space," Communications in Mathematical Physics, vol. 87, no. 4, pp. 577-588, 1983.

[3] J. Louko and S. N. Winters-Hilt, "Hamiltonian thermodynamics of the Reissner-Nordström-anti-de Sitter black hole," Physical Review D, vol. 54, no. 4, pp. 2647-2663, 1996.

[4] A. Chamblin, R. Emparan, C. V. Johnson, and R. C. Myers, "Holography, thermodynamics, and fluctuations of charged AdS black holes," Physical Review D, vol. 60, no. 10, Article ID 104026, 1999.

[5] R.-G. Cai, "Critical Behavior in Black Hole Thermodynamics," Journal of the Korean Physical Society, vol. 33, p. S477, 1998.

[6] R.-G. Cai and J.-H. Cho, "Thermodynamic curvature of the BTZ black hole," Physical Review D, vol. 60, Article ID 067502, 1999.

[7] A. Smailagic and E. Spallucci, "Feynman path integral on the non-commutative plane," Journal of Physics A, vol. 36, no. 33, pp. L467-L471, 2003.

[8] T. G. Rizzo, "Noncommutative inspired black holes in extra dimensions," Journal of High Energy Physics, no. 9, 2006.

[9] P. Nicolini, A. Smailagic, and E. Spallucci, "Noncommutative geometry inspired Schwarzschild black hole," Physics Letters B, vol. 632, no. 4, pp. 547-551, 2006.

[10] E. Spallucci, A. Smailagic, and P. Nicolini, "Non-commutative geometry inspired higher-dimensional charged black holes," Physics Letters B, vol. 670, no. 4-5, pp. 449-454, 2009.

[11] P. Nicolini, "Noncommutative black holes, the final appeal to quantum gravity: a review," International Journal of Modern Physics A, vol. 24, pp. 1229-1308, 2009.

[12] Y. S. Myung, Y.-W. Kim, and Y.-J. Park, “Thermodynamics and evaporation of the noncommutative black hole," Journal of High Energy Physics, no. 2, 2007.

[13] R. Banerjee, B. R. Majhi, and S. Samanta, "Noncommutative black hole thermodynamics," Physical Review D, vol. 77, no. 12, Article ID 124035, 2008.

[14] R. Banerjee, B. R. Majhi, and S. K. Modak, "Noncommutative Schwarzschild black hole and area law," Classical and Quantum Gravity, vol. 26, no. 8, 2009.

[15] K. Nozari and S. H. Mehdipour, "Hawking radiation as quantum tunneling from a noncommutative Schwarzschild black hole," Classical and Quantum Gravity, vol. 25, no. 17, Article ID 175015, 2008.

[16] G. Ruppeiner, “Thermodynamics: a Riemannian geometric model," Physical Review A, vol. 20, p. 1608, 1979.

[17] G. Ruppeiner, "Stability and fluctuations in black hole thermodynamics," Physical Review D, vol. 75, Article ID 024037, 2007.

[18] F. Weinhold, "Metric geometry of equilibrium thermodynamics," Journal of Chemical Physics, vol. 63, p. 2479, 1975.

[19] F. Weinhold, "Metric geometry of equilibrium thermodynamics. II. Scaling, homogeneity, and generalized Gibbs-Duhem relations," Journal of Chemical Physics, vol. 63, p. 2484, 1975.

[20] H. Quevedo, "Geometrothermodynamics," Journal of Mathematical Physics, vol. 48, no. 1, Article ID 013506, 2007.
[21] Y. S. Myung, Y.-W. Kim, and Y.-J. Park, "Quantum cooling evaporation process in regular black holes," Physics Letters B, vol. 656, no. 4-5, pp. 221-225, 2007.

[22] W. Kim, E. J. Son, and M. Yoon, “Thermodynamic similarity between the noncommutative Schwarzschild black hole and the Reissner-Nordström black hole," Journal of High Energy Physics, no. $4,2008$.

[23] H. Quevedo, "Geometrothermodynamics of black holes," General Relativity and Gravitation, vol. 40, no. 5, pp. 971-984, 2008.

[24] H. Quevedo and A. Sánchez, "Geometric description of BTZ black hole thermodynamics," Physical Review D, vol. 79, no. 2, Article ID 024012, 2009.

[25] H. Quevedo, A. Sánchez, S. Taj, and A. Vázquez, "Phase transitions in geometrothermodynamics," General Relativity and Gravitation, vol. 43, no. 4, pp. 1153-1165, 2011.

[26] P. C. W. Davies, "Thermodynamic theory of black holes," Proceedings of the Royal Society A, vol. 353, no. 1975, pp. 499521, 1977.

[27] P. C. W. Davies, “Thermodynamics of black holes," Reports on Progress in Physics, vol. 41, p. 1313, 1978.

[28] J. D. Brown, J. Creighton, and R. B. Mann, “Temperature, energy, and heat capacity of asymptotically anti-de Sitter black holes," Physical Review D, vol. 50, no. 10, pp. 6394-6403, 1994. 

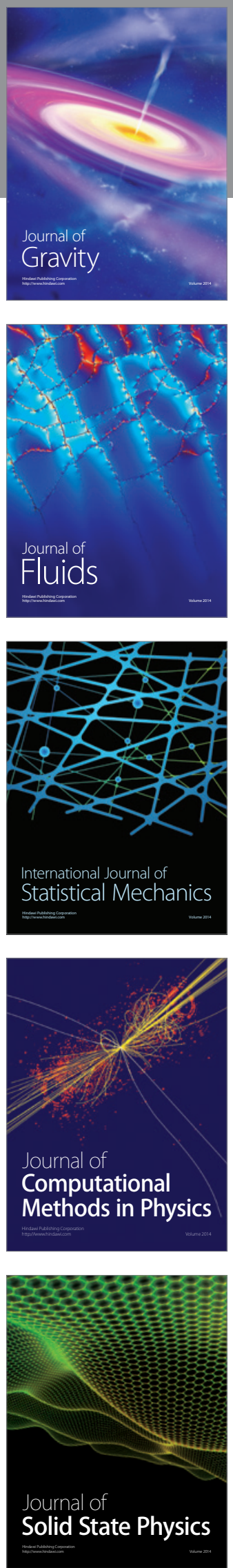

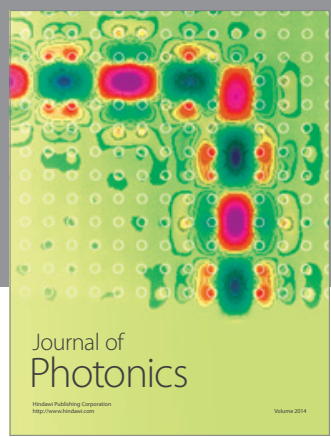

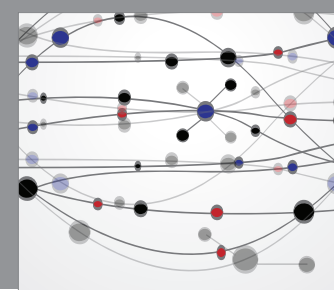

The Scientific World Journal

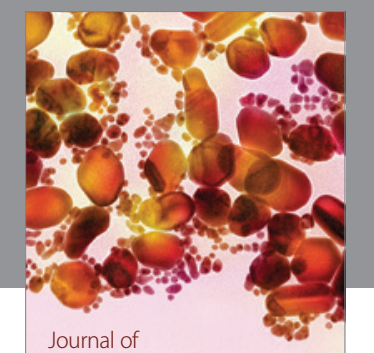

Soft Matter
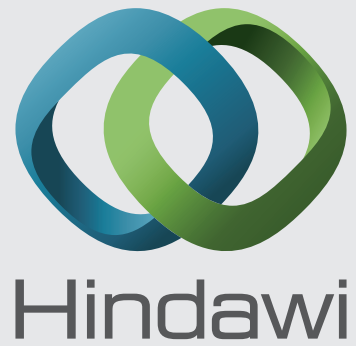

Submit your manuscripts at

http://www.hindawi.com
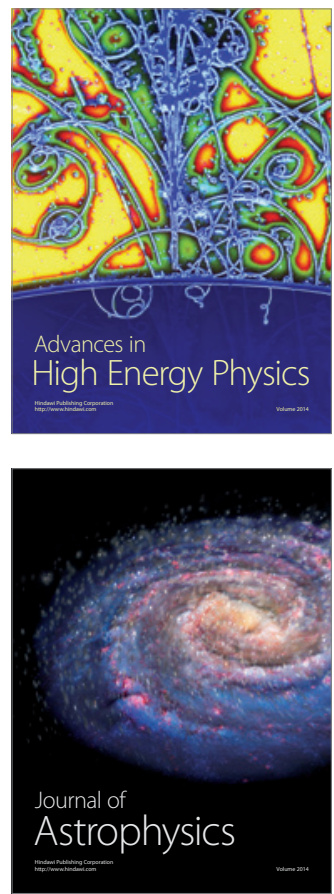
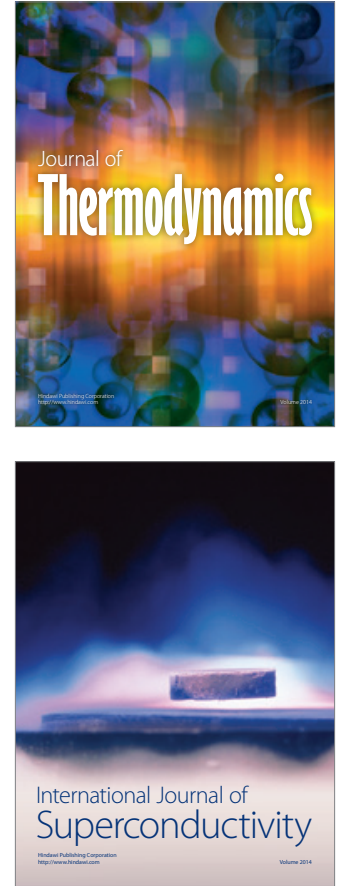
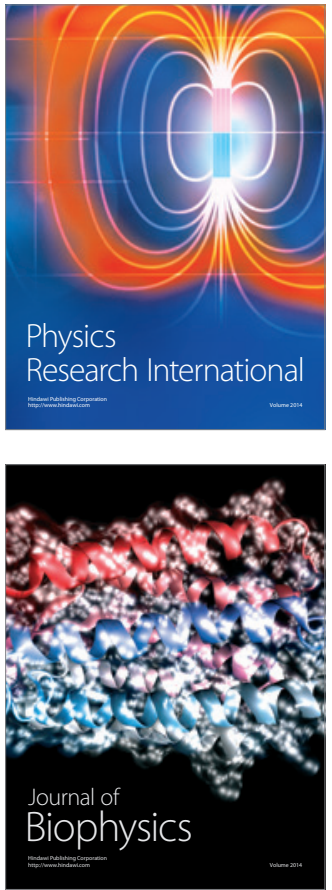
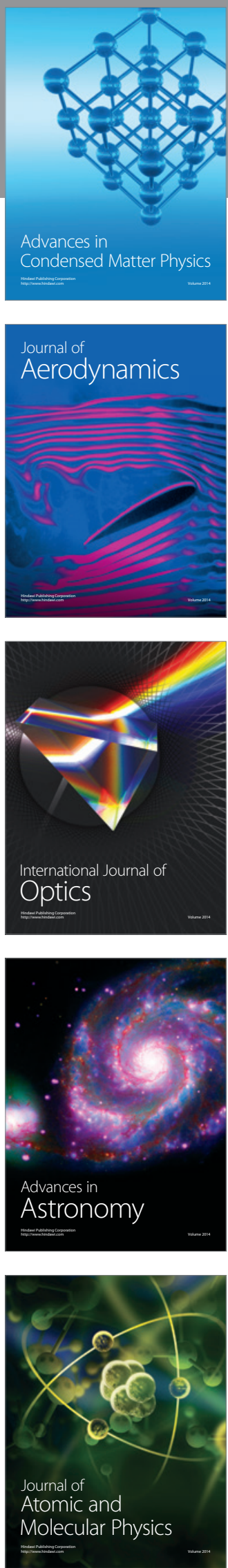